\title{
Expériences vécues d'infirmières œuvrant à titre de soignantes auprès d'un de leurs proches atteint de cancer avancé
}

par Lisa Cicchelli et Deborah McLeod

Quel honneur que de recevoir le Prix de conférence à la mémoire de Helene Hudson 2011. Les contributions de Helene Hudson aux soins infirmiers en oncologie ont eu un profond retentissement sur les soins en cancérologie au Canada. Il est manifeste que son dévouement envers l'oncologie a été d'une grande inspiration pour le personnel infirmier et qu'il a orienté les soins prodigués aux patients atteints de cancer et à leur famille. Nous remercions l'ACIO de nous avoir décerné cet honneur.

\section{Abrégé}

Il existe peu de travaux de recherche qui portent sur les expériences des infirmières prenant soin de membres de leur propre famille diagnostiqués de cancer. Afin de combler cette lacune, une démarche phénoménologique de type herméneutique a été utilisée en vue d'explorer les expériences vécues de cinq infirmières s'occupant de membres de leur famille atteints de cancer avancé. Leurs expériences révélaient un grand nombre de tensions et de conflits alors qu'elles tentaient de concilier les rôles d'infirmière et de soignante naturelle. Du plus profond de leurs expériences se dégageait le sentiment d'être prisonnières d'une toile faite d'attentes contradictoires. Leurs conflits en matière d'attentes provenaient de l'anticipation qu'elles avaient de la trajectoire de la maladie, des attentes de leur propre famille, des attentes des autres professionnels de la santé et des attentes que les infirmières avaient d'ellesmêmes en tant que soignantes naturelles. Les conflits entre leur vie professionnelle et leur vie personnelle leur posaient les plus grands défis. Les répercussions de cette situation de prestation de soins sont décrites.

\section{Au sujet des auteures}

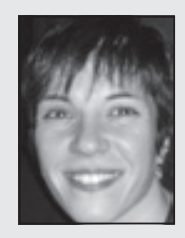

Lisa Cicchelli, inf., M.Sc.inf., infirmière coordonnatrice, Santé du sein, Centre de soins IWK-Santé du sein, Halifax, N.-É.

Tél. (bureau): (902) 470-2747; Téléc.: (902) 470-6696; Courriel: lisa.cicchelli@iwk.nshealth.ca

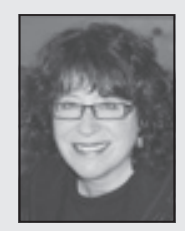

Deborah McLeod, inf., Ph.D., Centre des sciences de la santé Reine Elizabeth II, Cancer Care Program, Halifax, N.-É.

Tél. (bureau): (902) 473-2964; Téléc.: (902) 473-2965; Courriel: deborahl.mcleod@cdha.nshealth.ca
À un moment ou à un autre de leur vie, la plupart des infirmières assumeront des responsabilités en matière de prestation de soins auprès de membres de leur famille tels que partenaire de vie, parents vieillissants et autres proches qui sont malades. Comme il est prévu que les taux de cancer grimpent en flèche au cours des prochaines décennies, beaucoup d'infirmières seront amenées à prendre soin de proches diagnostiqués d'un cancer. Le cancer est une maladie complexe qui peut éventuellement déclencher une crise émotionnelle, pratique et existentielle chez l'individu concerné par le diagnostic et chez les personnes directement impliquées dans ses soins (Mills \& Aubeeluck, 2006). Lorsque le cancer parvient à son stade avancé, les soignants naturels éprouvent souvent un stress émotionnel et psychologique. Si les soignants naturels sont des infirmières, il est possible qu'elles pénètrent dans un monde qu'elles connaissaient uniquement par l'intermédiaire des patients et des familles dont elles s'étaient occupées auparavant. On en sait fort peu sur les expériences et les perspectives des infirmières qui prennent soin d'êtres chers diagnostiqués du cancer. Cette étude avait pour but d'explorer les expériences vécues des infirmières relativement à la prestation des soins lorsqu'un membre de leur famille est diagnostiqué d'un cancer avancé. Aux fins de la présente étude, le terme cancer avancé se rapporte à tout cancer parvenu à un stade évolutif, métastatique ou terminal.

\section{Contexte}

L'effet d'un diagnostic de cancer sur les proches du malade est bien décrit dans la littérature (Björk, Wiebe \& Hallström, 2005; Eldredge et al., 2006; Osse, Vernooij-Dassen, Schade \& Grol, 2006;

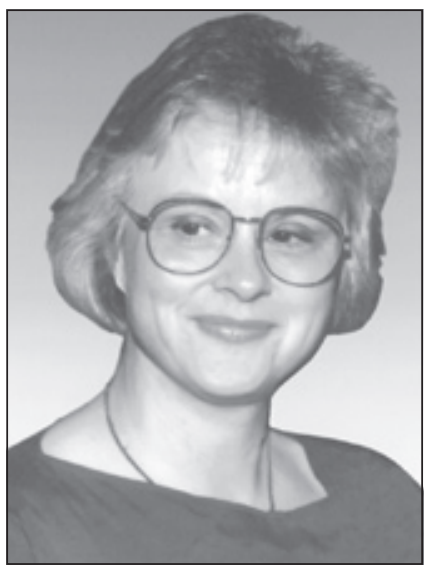

Helene Hudson-1945-1993 Schumacher et al., 2006; Scott, Hwang \& Rogers, 2006). Il a été démontré que le stress lié à la prestation des soins informels entraîne des niveaux élevés de détresse tels que la dépression, la colère, l'anxiété, la perturbation des relations et l'isolement (Koerner \& Kenyon, 2007; Perreault et al., 2004).

Il y a, au Canada, une demande croissante de ressources pour soigner les personnes âgées et les malades chroniques (Caron \& Bower, 2003; Nevidjon, 2004; O'Brien-Pallas et al., 2003). Les membres de la famille constituent un bassin important de 
personnes aptes à prendre soin des malades et des personnes âgées. Le profil type du soignant naturel est en fait une femme âgée de 46 ans, mariée, détentrice d'un emploi à plein temps qui consacre 18 heures par semaine à la prestation de soins informels (Lockwood, 2003). On se rend de plus en plus compte que des maladies comme le cancer sont devenues des enjeux familiaux plutôt que de concerner uniquement la personne touchée par la maladie (Caron \& Bowers, 2003; Dumont et al., 2006).

Certaines études précisent que, souvent, les tâches et les fardeaux des infirmières liés à la prestation des soins informels sont nombreux, sont variés et changent fréquemment tout au long de l'évolution de la maladie du proche concerné, tout comme c'est d'ailleurs le cas pour les soignants naturels pris dans leur ensemble (Mills \& Aubeeluck, 2006). Cependant, on comprend encore mal les différences qui peuvent exister entre les soignants naturels sans formation en santé et les infirmières qui fournissent des soins informels à un membre de leur famille. Une compréhension plus approfondie des enjeux pourrait aider à dégager des moyens possibles de soutenir les infirmières dans leurs rôles de soignantes naturelles, y compris des aménagements au niveau du travail. Des chercheurs et des organisations font remarquer que le système de santé commence à avoir de la difficulté à relever le défi que constitue l'incidence du vieillissement du personnel infirmier (Andrews, Manthorpe \& Watson, 2005; CRNNS, 2006; Villeneuve \& MacDonald, 2006). Par contre, aucune étude ne s'est, jusqu'à présent, fixé comme objectif d'explorer et d'aborder expressément la question des infirmières vieillissantes adoptant des rôles de prestation de soins auprès de proches.

\section{Méthodes}

Cette étude faisait appel à la phénoménologie herméneutique, une approche examinant le monde humain tel qu'il est éprouvé. La phénoménologie herméneutique cherche à découvrir la signification d'un phénomène particulier à titre d'expérience essentiellement humaine et ce, dans le contexte social, culturel et politique au sein duquel les expériences surviennent (van Manen, 1997).

L'approbation déontologique a été obtenue auprès du comité d'éthique de la recherche local. Les participantes à l'étude étaient des infirmières œuvrant en Nouvelle-Écosse qui prenaient en charge un aspect quelconque des soins d'un membre adulte de leur famille atteint d'un cancer avancé ou l'avaient fait avant que celui-ci ne décède de son diagnostic de cancer. Il n'était pas exigé que les soignantes naturelles vivent dans le même foyer que le proche malade, mais elles devaient habiter à proximité et fournir un aspect ou un autre des soins physiques ou émotionnels et participer activement à la prise de décision en matière de traitement. Afin d'assurer la confidentialité, des pseudonymes ont été attribués aux participantes et utilisés tout au long de l'étude. L'échantillon de l'étude se composait de cinq infirmières qui avaient toutes, à l'exception d'une seule, 45 ans ou plus. La plupart d'entre elles prenaient soin de parents ou de beaux-parents malades tandis que l'une d'elles s'occupait de sa tante. Les diagnostics des proches concernaient différents types de cancers: cancer du pancréas, du colon, du poumon, du sein et, lymphome.

Pour cette étude, les entrevues semi-structurées constituaient la principale méthode de collecte des données. Elles donnaient aux participantes la possibilité de parler de leurs expériences en matière de prestation de soins informels. Ces entrevues faisaient l'objet d'un enregistrement sonore, d'une transcription et d'une vérification de leur exactitude. Les transcriptions ont ensuite été lues à de multiples reprises, et des notes explicatives ont été rédigées. Les motifs thématiques ont alors été cernés.

\section{Résultats: Prises entre les deux}

Le thème général était le sentiment d'être prises entre les deux. Les infirmières décrivaient comment elles négociaient continuellement les limites et les attentes de leurs deux rôles - celui de membre de la famille et celui d'infirmière. Ce thème général comprend les sous-thèmes suivants: Concilier les limites professionnelles et personnelles, Détenir une information privilégiée, Faire l'expérience du système de soins du point de vue des soignés, Être témoin des effets de la maladie et enfin, Prendre des décisions au nom du proche malade.

\section{Attentes en matière de prestation:}

\section{Concilier les limites professionnelles et personnelles}

Selon les descriptions qu'en faisaient les infirmières participantes, la prestation de soins informels peut parfois s'avérer être une expérience frustrante. Quoiqu'elles décrivent souvent la confiance et l'aise avec lesquelles elles fournissaient des soins physiques, il leur arrivait parfois de ne pas se sentir à la hauteur et d'éprouver de l'impuissance face à leur rôle de prestation de soins informels. Cela était particulièrement vrai lorsqu'elles étaient témoins de souffrances émotionnelles ou spirituelles chez leur proche malade. Une répondante décrivait la difficulté qu'elle avait au niveau de son attente qui voulait qu'elle soit capable d'aider sa mère relativement à ses besoins émotionnels et «d'établir une communication thérapeutique».

Je crois que l'aspect le plus pénible de la prestation des soins informels [à ma mère] était les questions psychologiques. En tant qu'infirmière, je [savais] que les gens passent par toutes ces étapes du mourir. Ils éprouvent tour à tour déni, colère et frustration. Je voulais être capable de lui parler de ces questions. Mais elle ne voulait pas les aborder avec moi. Je me demandais "mais qu'est-ce qui cloche dans mon approche?» Je savais qu'elle était terrifiée. Je voulais lui donner une belle mort, une mort pleine de dignité, une mort dans le cadre de laquelle elle pourrait parler de ses sentiments et pour laquelle nous pourrions lui apporter de l'aide. Mais cela ne s'est pas produit comme ça. S'il y avait une chose que je pouvais changer, ce serait ça.

Une autre participante qui prenait soin de son père décrivait ainsi son expérience à ce même égard.

Je trouvais cela extrêmement frustrant parce qu'il n'y avait rien que je puisse faire pour l'aider. Je ne savais pas ce qu'il ressentait. Tu donnes un bain à quelqu'un et c'est fini. Cela peut constituer un défi physique. Cela peut être difficile. Mais le côté émotionnel, il est continuellement là et tu ressasses ça continuellement.

Aucune des participantes qui signalaient ces difficultés ne semblait remettre en question la pertinence de ses efforts visant à aborder les besoins émotionnels de son proche à la lumière de son expérience professionnelle ni ne se rendait compte du conflit inhérent à son double rôle - d'être cher et de professionnelle de la santé - dont elle était prisonnière. Ce double rôle créait des attentes et des obligations conflictuelles difficiles à gérer au beau milieu des émotions et du chagrin qui les assaillaient.

\section{Attentes en matière de prestation:}

\section{Détenir de l'information privilégiée}

Les infirmières ont des connaissances professionnelles qui les aident à comprendre diverses maladies ainsi que les soins et les traitements requis. Les membres de leur famille voient souvent en elles des sources d'information pouvant aider la famille à surmonter la maladie. Les infirmières de cette étude avaient divers degrés de connaissance de l'oncologie, mais il arrivait parfois qu'elles ne connaissent pas ce domaine aussi bien que les membres de la famille s'y attendaient. D'autres fois, c'étaient les connaissances que l'infirmière possédait qui faisaient empirer ses craintes et son stress. 
Je ne sais pas si je n'aurais pas été aussi effrayée sans mes antécédents en soins infirmiers. D'un côté, c'était bien d'avoir toutes ces connaissances, mais d'un autre, j'avais les antécédents qui me permettaient de voir et de savoir ce qui pouvait bien être en train de se produire. Aujourd'hui, quand je regarde en arrière, je crois que c'était utile, mais je pense que les choses étaient un peu plus stressantes parce que je savais que la mort pouvait survenir.

Les infirmières participant à l'étude détenaient chacune différents types de connaissances à propos de la situation de leur proche malade. Dans certains cas, elles y voyaient un atout qui leur permettait de mieux comprendre la situation et de fournir du soutien aux autres membres de la famille. Dans d'autres cas, cela représentait une source de stress, notamment lorsqu'elles détenaient de l'information "privilégiée» qui leur avait été transmise par d'autres professionnels de la santé parce qu'elles étaient infirmières, de l'information qui n'était habituellement pas partagée avec la famille. Dans ces cas-là, certaines d'entre elles ne savaient pas comment ou bien quand elles devaient partager cette information avec les autres membres de la famille.

\section{Faire l'expérience du système de}

\section{soins du point de vue des soignés}

Se retrouver bénéficiaire de soins plutôt que prestataire constituait tout un défi pour certaines des participantes. Pour quelquesunes d'entre elles, il s'agissait de leur toute première expérience de bénéficiaire de soins. Leurs expériences en tant que proches de malades étaient souvent en contradiction avec leurs attentes relatives aux professionnels et systèmes de santé, ce qui créait des tensions. Une infirmière a offert le commentaire suivant à cet effet:

Les intervenants des soins palliatifs ont fait une évaluation à domicile. Je leur avais signalé que, selon moi, elle [ma mère] était déprimée. Ils lui ont prescrit un médicament à faible dose mais celui-ci n'a eu aucun effet. Je leur ai dit qu'elle était déprimée. Je pensais qu'ils allaient changer son ordonnance quand ils viendraient lui parler. Ils lui ont demandé: «Êtes-vous déprimée?» et elle a répondu "Non», et cela n'a pas été plus loin. Est-ce comme ça qu'on réalise une évaluation psychologique en vue de dépister la dépression? [Ils] ne vivent pas avec elle à longueur de journée et j'étais bouleversée qu'ils ne m'aient pas écoutée.

Une participante a décrit les émotions qu'elle ressentait en réaction à la manière dont elle avait été traitée à titre de membre de la famille.

[J'étais] furieuse. L'idée de traiter le patient dans sa globalité ne s'arrête pas au seul patient. Il faut inclure la famille également. Je ne crois pas qu'ils aient fait du bon travail. Ma mère était la cible de leurs soins et ils ne se souciaient de personne d'autre... je pense que cela se limitait aux soins physiques. Il n'y avait aucune composante psychologique.

Les attentes en matière de soins n'étaient pas toujours satisfaites et, dans ces cas-là, il arrivait souvent que les infirmières-aidantes naturelles comblent les lacunes en faisant appel à leurs relations professionnelles. "Je savais exactement qui appeler... Nous avons fait venir quelqu'un des soins à domicile, quelqu'un de VON et il y a avait une troisième personne. » La répondante a continué en décrivant la colère qu'elle ressentait vis-à-vis de la manière dont les professionnels de la santé partageaient l'information entre eux.

Cela devenait tannant... J'en suis devenue vraiment furieuse.

Je ne cessais de me dire qu'il était impossible d'excuser le fait que trois personnes posent la même série de questions le même jour. Ça n'avait pas d'allure. Comment peut-on réaliser une évaluation et ne pas le faire savoir aux autres?

\section{Être témoin des effets de la maladie}

Certaines des infirmières, tout comme les membres de la famille sans antécédents en soins infirmiers, éprouvaient de la détresse lorsqu'ils constataient les effets de la maladie. Quoique toutes les participantes aient déjà été témoins du déclin de patients et de leur décès du fait de la maladie, il est manifeste qu'il s'agit d'une toute autre expérience lorsque cela concerne un être cher. Elles essayaient d'aider à tout prix car elles jugeaient qu'elles devraient être capables de soulager la souffrance.

Je lui ai donné des occasions de parler de ses sentiments et de sa crainte de mourir. Elle ne voulait pas aborder quelque sentiment que ce soit. [J'ai dû la regarder] en train de souffrir et de fixer les yeux sur le mur. [Cela était] difficile à supporter. Elle venait à table, mangeait deux fois rien, ne disait pas un seul mot, puis s'en allait. Il est pénible de voir l'état d'une personne décliner comme ça et de ne pas savoir ce qu'elle ressent... Je ne pouvais pas [supporter] de la voir souffrir. [La mettre] dans la baignoire, lui enlever ses vêtements, voir le squelette qu'elle était. Elle n'avait plus que la peau et les os. C'était fort pénible.

\section{Prendre des décisions au nom du proche malade}

Les infirmières de l'étude devaient fréquemment participer à la prise de décisions complexes sur des sujets tels que les traitements anticancéreux. Une infirmière a décrit le tourment personnel que lui causait la décision de son proche de subir un traitement de chimiothérapie. L'expérience préalable de l'infirmière en soins palliatifs avait façonné l'opinion qu'elle avait de ce type de traitement. Elle convenait qu'au départ elle n'était pas d'accord avec cette décision, mais elle a concédé plus tard que la chimiothérapie avait été bénéfique pour son être cher.

[J'étais là] pour aider au niveau de la prise de décision, d'une part... et même après la première séance, ils n'étaient pas sûrs au début si elle devait recevoir autre chose que de la radiothérapie pour ses métastases osseuses et le contrôle de la douleur... Cela a été un véritable débat intérieur parce que je vois tant de patients souffrant des effets de la chimio et subissant de la chimio qu'ils ne devraient probablement pas subir... c'est vraiment dur de savoir si cela cause plus de problèmes ou pas. Et c'est ce que nous constatons, quand nous prodiguons nos soins, il est certain que je constate des avantages également, mais une chose est sûre, je ne voulais pas lui faire subir des choses pires que ce qu'elle avait subi jusque-là... Cela me déchirait vraiment à l'intérieur. Mais elle s'en est fort bien tirée, n'a pas eu d'effets secondaires marqués et a même commencé à se sentir mieux dans les jours suivants... Nous sommes passés de l'absence de tout espoir au plein espoir.

\section{Discussion}

Cette étude était conçue pour explorer les expériences vécues des infirmières prenant soin d'un de leurs proches atteint de cancer. Ses résultats jettent de la lumière sur les tensions et les enjeux auxquels les infirmières sont confrontées tandis qu'elles tentent de concilier le double rôle d'infirmière et de soignante naturelle. Quoique que nous ayons constaté que les infirmières de l'étude avaient certaines expériences de prestation de soins informels en commun avec les autres soignants naturels, elles signalaient également des expériences qui les démarquaient de ces derniers du fait de leur profession.

Il est possible que pour ce qui est de la prestation de soins informels, les attentes impliquent une anticipation des indices ou des prédictions sur la trajectoire de la maladie, sur les rôles assumés par les soignants naturels et par les patients et sur le comportement des patients et des membres de leur famille. Les infirmières participant à l'étude avaient des attentes particulières relativement à leur rôle de soignante naturelle du fait de leurs 
connaissances professionnelles. Il leur arrivait souvent d'anticiper les phases suivantes de la maladie et elles essayaient de s'y préparer ainsi que leurs proches. Mills et Aubeeluck (2006) ont également constaté que les infirmières de leur étude parlaient fréquemment de "se projeter dans le temps» et d'anticiper les phases suivantes de la maladie parce qu'elles savaient déjà ce à quoi s'attendre.

Confrontées comme elles l'étaient au besoin de prodiguer des soins et aux puissantes attentes de la famille, les infirmières de notre échantillon assumaient de bon cœur leurs rôles de prestataires de soins informels. Elles décrivaient les longues heures qu'elles y consacraient, les conversations difficiles avec les membres de la famille, les nombreux trajets pour être auprès de leur être cher ainsi que le stress et le fardeau que cela représentait. Leurs expériences étaient épuisantes sur le plan physique et émotionnel, ce qui faisait écho aux descriptions des autres soignants naturels (Mills \& Aubeeluck, 2006, Scott et al., 2006). Les participantes assumaient le rôle - exigeant - de prestataire de soins informels, tout en poursuivant en grande partie leurs fonctions professionnelles. Selon leurs descriptions, elles se sentaient obligées de dispenser des soins parce qu'elles exerçaient le métier d'infirmière et il est possible qu'elles se soient donné, ce faisant, un fardeau supplémentaire au titre de la prestation des soins informels (WardGriffin et al., 2004).

Bien qu'elles aient exprimé le stress qu'elles ressentaient du fait des attentes des membres de la famille vis-à-vis d'elles, les infirmières de cette étude n'ont jamais discuté de ces attentes ni ne les ont remises en question ou clarifiées. Au contraire, elles acceptaient le rôle de prestataire de soins informels sans discuter de ce qui était attendu d'elles. Aucune des infirmières ne semblait vouloir examiner l'incidence que le rôle de soignante naturelle pourrait avoir sur leur relation avec l'être cher. Clarifier les attentes dès les premiers temps du processus morbide pourrait constituer, pour les infirmières, une façon de gérer une situation particulièrement stressante. En clarifiant les attentes et en communiquant le plus tôt possible à ce propos, elles pourraient favoriser les opportunités de soutien mutuel et de communication transparente continuelle avec les autres membres de la famille. Ce faisant, le fardeau de prestation de soins informels pourrait être partagé avec quelqu'un d'autre plutôt que de répliquer l'idée fausse selon laquelle la prestation de soins informels est «facile» ou «naturelle» pour une infirmière, du simple fait de sa formation professionnelle.

Des études ont démontré que les infirmières prodiguant des soins informels sont fortement influencées par les normes et les attentes relatives à la fourniture de soins, le plus souvent sans que leurs propres besoins ne soient pris en compte (Mills \& Aubeeluck, 2006). Selon Grande et al. (2009), les normes de la prestation des soins informels s'inscrivent habituellement dans des contextes sociaux et culturels et sont en outre renforcées par les interactions avec les membres de la famille, le proche malade et par les émotions intériorisées des soignants. Les infirmières examinées dans le cadre de cette étude ont accepté le rôle en vertu de leurs liens familiaux et de leur situation sociale, mais leurs antécédents en soins infirmiers les définissaient et constituaient une puissante force qui façonnait leur expérience de prestation de soins informels.

Le rôle de prestataire de soins informels tel que joué par les infirmières n'était pas toujours décrit comme étant une expérience négative. Les infirmières se disaient généralement satisfaites de prendre soin de leurs proches et étaient en grande partie heureuses de leur implication. Les infirmières étaient fières de leur aptitude à dispenser des soins et à faire face aux défis; cela les remplissait d'un sentiment d'utilité et de valeur personnelle. Leurs connaissances infirmières ont aidé chacune d'elles à réconforter un être cher. Leur expérience de prestation de soins informels à un proche a également influencé et façonné leur propre pratique professionnelle.
Pour beaucoup de gens, la prestation de soins à un proche adulte atteint de cancer n'est pas un rôle qu'ils anticipent ni ne choisissent, et celui-ci est souvent assumé sans préavis ni formation préalable. La situation des infirmières est différente puisqu'elles ont fait des études et ont suivi une formation en soins de santé et peuvent prévoir la nature de la maladie et l'intensité du travail de prestation de soins informels. Dans l'esprit des participantes et dans celui des membres de leurs familles, il était évident qu'elles devaient jouer un rôle important dans la prestation des soins informels au proche malade, un choix qui est assurément moins évident dans les familles qui ne comprennent pas d'infirmière ou de professionnel des soins de santé.

Les limites entre la prestation de soins à titre personnel et à titre professionnel étaient floues et exigeaient des manœuvres continuelles de la part des infirmières ayant participé à cette étude. Détenir une information privilégiée était l'aspect le plus exigeant de la situation, qu'elles possèdent celle-ci du fait de leur formation ou du fait de l'avoir reçue d'autres professionnels de la santé en raison de leur condition d'infirmières. Comme elles étaient capables d'interpréter la situation au fur et à mesure de son développement, les infirmières avaient habituellement de l'information beaucoup plus tôt que tout autre membre de leur famille. Il leur était difficile de décider dans quelle proportion elles devaient la divulguer, de quelle manière et à quel moment.

Les soignants naturels, y compris ceux qui sont également des professionnels de la santé, passent souvent inaperçus dans les systèmes de santé (Mills \& Aubeeluck, 2006). Les infirmières participant à cette étude ont déclaré qu'elles souhaitaient recevoir une quantité donnée de soutien émotionnel et de soins de soutien de la part des professionnels de la santé prenant en charge le proche malade. Cependant, quelques-unes se sont déclarées déçues de la façon dont elles étaient traitées. Certaines ont dit qu'elles se considéraient comme étant des clientes dans le contexte de soins, mais qu'elles sentaient qu'on les ignorait ou qu'on ne s'occupait pas d'elles dans le cadre de leur expérience. Certaines des infirmières ne voulaient pas critiquer ouvertement leurs collègues œuvrant à titre professionnel, ce qui les a peut-être empêchées d'exprimer leur mécontentement et le conflit émotionnel entre elles et les professionnels de la santé dispensant des soins à leur proche malade.

Les infirmières appartenant à cette étude choisissaient de poursuivre l'expérience de prestation de soins informels dans presque toute son étendue et elles trouvaient l'expérience plutôt positive et enrichissante. Des études ont démontré que dans la plupart des cas il est préférable que les soignants naturels dispensant des soins informels à un proche diagnostiqué d'un cancer aient un emploi (Kim et al., 2006; Lefaiver et al., 2009). Le fait de travailler tout en dispensant des soins à ce proche peut présenter des occasions de répit aux soignants naturels, leur donner des occasions de recharger leurs ressources psychologiques et de se concentrer sur des choses autres que leurs responsabilités de soins dans la vie privée. On pourrait donc conclure que la décision prise par les infirmières de continuer leurs activités professionnelles a contribué à la satisfaction qu'elles ressentaient sur le plan de leur implication auprès de leur proche malade.

$\mathrm{Au}$ cours des dix dernières années, une attention croissante a été portée au travail des infirmières et à la nécessité d'aborder des enjeux liés à la conciliation travail-famille tels que la santé, le bienêtre et la souplesse des horaires. La maladie d'un proche peut créer de nouvelles tensions dans ce domaine étant donné que les infirmières essaient de jongler avec les exigences de leur emploi et celles de la prestation de soins informels. Les infirmières participantes ont trouvé utile de pouvoir prendre des congés pour être auprès du proche malade. En outre, elles ont souvent faire valoir à quel point la souplesse en milieu de travail venait appuyer leur capacité à prendre soin des proches atteints de maladie. La recherche confirme 
l'importance de la souplesse en milieu de travail afin d'assurer un juste équilibre entre les responsabilités professionnelles et les responsabilités familiales (Andrews et al., 2005; CRNNS, 2006; Scott et al., 2006). Cette souplesse pourrait inclure des mesures telles que la modification des horaires, la modification des tâches assignées et des heures de travail. Ce besoin revêt une importance croissance étant donné que les infirmières vieillissent et qu'il est plus probable qu'elles prennent soin de proches âgés et malades.

\section{Conclusion}

Les infirmières qui prodiguent des soins informels à des membres de leur famille se trouvent dans une situation originale puisqu'elles ont des connaissances sur la maladie et les ressources en soins de santé et une bonne confiance dans leur aptitude à dispenser des soins informels. Les infirmières ont formulé plusieurs enjeux relatifs à la prestation de soins informels qui sont également cernés par les autres soignants naturels. Elles décrivaient également des enjeux qui semblent propres aux infirmiers et infirmières assumant ce rôle, telles que leurs attentes en matière de soins, la divulgation de l'information privilégiée et le flou entourant les limites entre la vie personnelle et professionnelle, autant d'enjeux qui peuvent constituer un fardeau supplémentaire. Les résultats de cette étude indiquent qu'une compréhension plus approfondie de la dispensation de soins formels et informels par les infirmières pourraient les aider à surmonter les difficultés associées à la prise en charge informelle d'êtres chers.

\section{Références}

Andrews, J., Manthorpe, J., \& Watson, R. (2005). Employment transitions for older nurses: A qualitative study. Journal of Advanced Nursing, 51(3), 298-306. doi:10.1111/ j.1365-2648.2005.03501.x

Björk, M., Wiebe, T., \& Hallström, I. (2005). Striving to survive: Families lived experiences when a child id diagnosed with cancer. Journal of Pediatric Oncology Nursing, 22(5), 265-275. doi:10.1177/1043454205279303

Caron, D., \& Bowers, B.J. (2003). Deciding whether to continue, share or relinquish caregiving: Caregiving views. Qualitative Health Research, 13(9), 1252-1271. doi:10.1177/10497323032 57236

College of Registered Nurses of Nova Scotia (CRNNS). (2006). Retirement and Retention of Late Career Nurses in Nova Scotia. Halifax, Canada. Author.

Dumont, S., Turgeon, J., Allard, P., Gagnon, P., Charbonneau, C., \& Vezina, l. (2006). Caring for a loved one with advanced cancer: Determinants of psychological distress in family caregivers. Journal of Palliative Medicine, 9(4), 912-921. doi:10.1089/ jpm.2006.9.912

Eldredge, D.H., Nail, L., Maziarz, R.T. Hansen, L.K., Ewing, D., \& Archbold, P.G. (2006). Explaining family caregiving role strain following autologous blood and marrow transplantation. Journal of Psychological Oncology, 24(3), 53-74. doi:10.1300/ J077v24n03_03

Grande, G., Stajduhar, K., Aoun, S., Toye, C., Funk, L., AddingtonHall, J., Payne, S., \& Todd, C. (2009). Supporting lay carers in end of life care: Current gaps and future priorities. Palliative Medicine, 23, 339-344. doi:10.1177/0269216309104875

Kim, Y., Baker, F., Spillers, R.L., \& Wellisch, D.K. (2006). Psychological adjustment of cancer caregivers with multiple roles. Psycho-oncology, 15, 795-806. doi:10.1002/pon.1013

Koerner, S.S., \& Kenyon, D.B. (2007). Understanding "good days" and "bad days": Emotional and physical reactivity among caregivers for elder relatives. Family Relations, 56(1), 1-11. doi:10.1111/j.1741-3729.2007.00435.x

Lefaiver, C.A., Keough, V., Letizia, M., \& Lanuza, D.M. (2009). Quality of life in caregivers providing care for lung transplant candidates. Progress in Transplantation, 19(2), 142-52.

Lockwood, N.R. (2003). The reality of the impact of older workers and eldercare in the workplace. HR Magazine, 48(12), 1-11.

Mills, J., \& Aubeeluck, A. (2006). Nurses' experiences of caring for their own family members. British Journal of Nursing, 15(3), 160-165.

Nevidjon, B. (2004). Managing from the middle: Integrating midlife challenges of children, elder parents, and career. Clinical Journal of Oncology Nursing, 8(1), 72-75. doi:10.1188/04.CJON.72-75

O’Brien-Pallas, L., Alksnis, C., \& Wang, S. (2003). Envisager l'avenir: Prévoir la retraite chez les IA au Canada. Ottawa: Institut canadien d'information sur la santé.

Osse, B.H., Vernooij-Dassen, M.J., Schade, E., \& Grol, R.P. (2006). Problems experienced by informal caregivers of cancer patients and their needs for support. Cancer Nursing, 29(5), 378-388.

Perreault, A., Fothergill-Bourbonnais, F., \& Fiset, V. (2004). The experience of family members caring for a dying loved one. International Journal of Palliative Nursing, 10(3), 133-143.

Schumacher, K.L., Beidler, S.M., Beeber, A.S., \& Gambino, P. (2006). A transactional model of cancer family caregiving skill. Advances in Nursing Science, 29(3), 271-286.

Scott, L.D., Hwang, W.T., \& Rogers, A.E. (2006). The impact of multiple caregiving roles on fatigue, stress, and work performance among hospital staff nurses. Journal of Nursing Administration, 36(2), 86-95.

van Manen, M. (1997). Researching Lived Experience. London: University of Western Ontario.

Villeneuve, M., \& MacDonald, J. (2006). Vers 2020: Visions pour les soins infirmiers. Ottawa: Association des infirmières et infirmiers du Canada.

Ward-Griffin, C. (2004). Nurses as caregivers of elderly relatives: Negotiating personal and professional boundaries. Canadian Journal of Nursing Research, 36(1), 92-114.

Williams, L.A. (2007). Whatever it takes: Informal caregiving dynamics in blood and marrow transplantation. Oncology Nursing Forum.,34(2), 379-387. doi:10.1188/07.ONF.379-387 